\title{
Research on ecological restoration of rural landscape based on environmental value-added
}

\author{
Ajun Wan ${ }^{1, *}$, Xiaolei $\mathrm{Qi}^{1}$, Runqiu Tu${ }^{1}$ \\ ${ }^{1}$ Institute of Modern Agricultural Science and Engineering, Tongji University, Shanghai, China
}

\begin{abstract}
Reasonable planning of green space can effectively play the urban ecosystem service function and promote the sustainable development of rural areas. Taking LiuTian village in Shaanxi Province as an example, based on the analysis of rural landscape pattern, this paper explores the landscape comprehensive evaluation and planning ideas based on environmental value-added. The research results provide reference for the landscape construction and planning of the same type of villages in Guanzhong area of Shaanxi Province, and have good application prospects.
\end{abstract}

\section{Introduction}

In recent years, with the rapid development of new rural construction, the accompanying ecological problems have become increasingly apparent. Planning first is an important measure to revitalize the countryside. Therefore, it is of great theoretical significance and practical value to put forward effective planning and design methods and schemes for the construction of new socialist countryside. Based on ecological and social considerations, Iiyama, $\mathrm{N}$ and others in Japan established a framework to evaluate rural landscape resources from the perspective of landscape ecology ${ }^{[1]}$. Assumma, V and others in Italy proposed a comprehensive evaluation method to measure the attractiveness of Rural Landscape ${ }^{[2]}$. Sun et al. Of South Korea evaluated the aesthetic value of mountain landscape and predicted the development trend of mountain landscape by using geospatial information system and artificial neural network ${ }^{[3]}$. Kovacs, KF and others summarized most of the services and functions of rural landscape through the application analysis of landscape indicators, and evaluated its entertainment, cultural and educational functions, aesthetic functions and other aspects by taking Tosas hansag and rabakoz as examples ${ }^{[4]}$. At the same time, the content of domestic landscape evaluation can be roughly divided into three categories ${ }^{[5]}$. One is the overall evaluation of rural landscape. Liu binyi put forward the evaluation index system of rural landscape oriented by human settlement environment in five aspects of rural landscape habitability, accessibility, compatibility, sensitivity and beauty ${ }^{[6]}$; the second is landscape ecological evaluation. Xiao $\mathrm{He}$ et al. Established a small-scale landscape database, focused on the landscape spatial structure and attributes, conducted ecological evaluation of rural landscape, identified the main ecological environment problems of rural landscape construction ${ }^{[7]}$; the third is rural landscape resource evaluation. Based on the characteristics of rural landscape, Xie Hualin constructed a hierarchical evaluation index system of rural landscape aesthetic feeling, and applied the proposed fuzzy comprehensive evaluation model to Baijiatuan Village, Wenquan Town, Haidian District, Beijing ${ }^{[8]}$.

In view of the dual constraints of rural development and environment, the academic circles have carried out a lot of relevant research, but these cases are still lack of systematic, comprehensive and quantitative research from evaluation to planning process, and lack of research on the combination of theory and design practice. Taking LiuTian village in Weinan City of Shaanxi Province as an example, this paper constructs the rural landscape planning method system and specific planning scheme based on the theory of environmental value-added.

\section{Materials and methods}

\subsection{Theoretical system of rural landscape comprehensive planning based on environmental value-added}

The rural landscape comprehensive planning system based on environmental value-added takes rural landscape planning as the starting point, landscape ecology and environmental value-added as the theoretical basis, and modern agriculture and rural tourism as the development orientation, and puts forward the rural planning method system (as shown in Figure 1). At the same time, this chapter puts forward the landscape planning scheme of LiuTian village, in order to enhance the landscape value of the village, optimize the layout of characteristic industries, enhance the comprehensive index of regional landscape, and realize value-added development. 


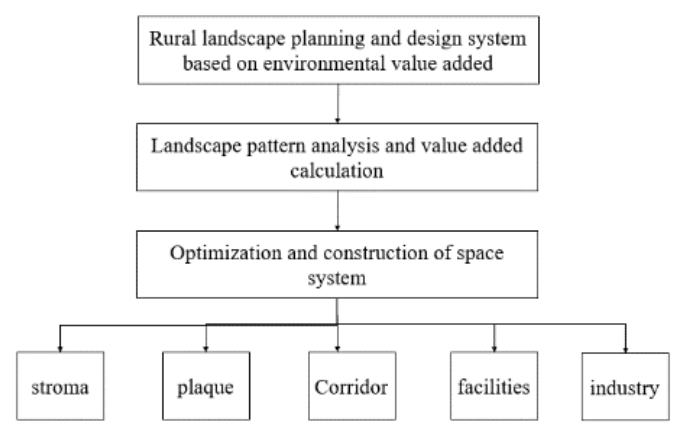

Figure 1. Structure chart of rural landscape planning and design method based on environmental value added

\subsection{Overview of the research area}

LiuTian village is located in the middle of Shaanxi Province, which is a suburban village with superior geographical location. LiuTian village has four distinct seasons: cold, warm, dry and wet, with sufficient sunshine, water and heat in the same season, and large interannual variation of temperature and precipitation. It is a warm temperate semi humid and semi-arid monsoon climate. Its geographical location is superior. It is close to Weinan City and the provincial capital Xi'an. The main internal roads are asphalt roads, and a small number of roads are earth roads that need to be improved. The village area is about $200 \mathrm{mu}$, and the cultivated land area is about $1660 \mathrm{mu}$. There is no idle land. The cultivated products include wheat, rice, grape, watermelon, jujube, etc. There are more than 170 households and 705 people in the village. The terrain of the village is relatively flat, without commanding heights such as mountains. At the same time, LiuTian village is located in the important tourism line of Weinan City, and the surrounding tourism resources are rich.

\subsection{Data source and preprocessing}

In this study, GIS visual interpretation method was used to extract landscape elements of LiuTian village, then FRAGSTATS technology was used to calculate the landscape pattern index, and ecological significance was used to analyze the landscape pattern of LiuTian village.

\subsection{Determination of landscape classification system}

The landscape information of LiuTian village was extracted and classified by GIS visual interpretation. The landscape types of LiuTian village are divided into 11 categories: rural residential land, industrial and mining land, public facilities land, cultivated land, garden land, facilities agricultural land, landscape and special land, water area, grassland, field road and rural road.

\subsection{Selection of landscape pattern index}

Landscape index is a common analysis method in landscape ecology, which can effectively reflect the landscape pattern information. By fully analyzing the ecological significance of each landscape index and referring to the research results of domestic and foreign scholars, this paper selects 9 indexes and calculates the data by FRAGSTATS.

\section{Results and analysis}

\subsection{Analysis of landscape patch characteristics}

There are 11 kinds of landscape types in LiuTian village, and the area of different landscape types is quite different. Among them, the landscape area of cultivated land is $110.95 \mathrm{hm} 2$, which is the absolute advantage, while the natural ecological patch area such as grassland is seriously lacking, which is only $0.45 \mathrm{hm} 2$. The area of cultivated land is the absolute advantage, with high aggregation degree, large patch cohesion and good natural connectivity.

Table 1. The analysis of village patch structure characteristics.

\begin{tabular}{|c|c|c|c|c|c|c|c|}
\hline $\begin{array}{l}\text { Landscape } \\
\text { types }\end{array}$ & $\mathrm{NP}$ & $\mathrm{CA} / \mathrm{hm} 2$ & PLAND $/ \%$ & PD & CONTAG & LSI & LPI/\% \\
\hline Cultivated land & 24 & 110.95 & 80.36 & 17.38 & 99.68 & 6.56 & 16.21 \\
\hline Wasteland & 23 & 4.34 & 3.14 & 16.66 & 98.13 & 18.44 & 0.37 \\
\hline waters & 2 & 1.57 & 1.14 & 1.45 & 99.23 & 16.51 & 1.03 \\
\hline Field Road & 10 & 2.6 & 1.89 & 7.24 & 98.43 & 36.31 & 0.4 \\
\hline garden plot & 8 & 5.17 & 3.75 & 5.79 & 98.9 & 3.72 & 1.38 \\
\hline $\begin{array}{l}\text { Land for facility } \\
\text { agriculture }\end{array}$ & 1 & 0.67 & 0.49 & 0.72 & 98.86 & 1.79 & 0.49 \\
\hline $\begin{array}{l}\text { Landscape } \\
\text { architecture and } \\
\text { special land }\end{array}$ & 3 & 0.45 & 0.32 & 2.17 & 97.52 & 2.3 & 0.13 \\
\hline $\begin{array}{l}\text { Land for rural } \\
\text { residents }\end{array}$ & 18 & 10.14 & 7.35 & 13.04 & 98.84 & 5.3 & 0.98 \\
\hline Rural road & 2 & 1.42 & 1.03 & 1.45 & 98.91 & 14.91 & 0.57 \\
\hline $\begin{array}{l}\text { Land for public } \\
\text { facilities }\end{array}$ & 6 & 0.28 & 0.2 & 4.35 & 96.2 & 2.31 & 0.12 \\
\hline $\begin{array}{l}\text { Industrial and } \\
\text { mining land }\end{array}$ & 2 & 0.46 & 0.33 & 1.45 & 98.01 & 1.9 & 0.2 \\
\hline
\end{tabular}

According to the analysis table of village patch structure characteristics (Table 1), cultivated land, grassland and rural residential land have the largest degree of landscape fragmentation. The number of patches NP is 24,23 and 18 respectively, and the patch density PD is $17.38,16.66$ and 13.04 respectively. The landscape is greatly disturbed by human activities, and the aggregation index AI of each landscape type is very high. The highest is cultivated land, with AI of 99.47. In addition, the landscape distribution in the region is uneven, with the sheei of 0.35 ; and the overall landscape diversity is not high, with the Shannon diversity index of 0.85 .

\subsection{Overall construction of landscape pattern and ecological restoration strategy}

Through the above analysis of the landscape pattern of LiuTian village, the landscape elements, characteristics and area proportion of LiuTian village are defined, and the planning and design method system and specific planning scheme are established.

(1) Greening disordered grassland. Through the greening of grassland, we can make up for the lack of 
grassland, woodland and other ecological service landscape defects in LiuTian village. For the most extensive agricultural planting (farmland, garden) matrix, on the basis of ensuring and improving the productivity of agricultural landscape, we should strengthen the improvement of other aspects of landscape. The construction of agricultural landscape is divided into general cultivated land landscape, characteristic cultivated land landscape and vegetable and fruit garden. On both sides of the central road and green corridor, special cultivated land landscape is arranged. The landscape construction of characteristic cultivated land adopts the reasonable collocation of various crops to reflect the four seasons color of different local crops. The planting space should be varied and clear-cut. At the same time, the viewing place should be set up for tourists to enter. The construction of vegetable and fruit garden landscape mainly includes the establishment of grape picking garden, walnut green planting base and other planting areas to form a compound multi variety leisure agricultural park; promote the diversified cultivation of local mustard, grape, walnut and other agricultural products to form a new pattern of vegetable and fruit agricultural industry with mustard planting as the core and grape, kiwi and other fruits as the main. In the aspect of general cultivated land landscape construction, we should actively introduce high-quality wheat varieties, introduce the latest cultivation techniques of wheat at home and abroad, such as soil testing, formulated fertilization, water-saving irrigation, integrated pest control, etc., so as to ensure a large area of flat planting area and form a good crown line and farmland edge. At the same time, on the basis of sightseeing, picking activities and farming experience days are provided to enhance the overall leisure tourism atmosphere.

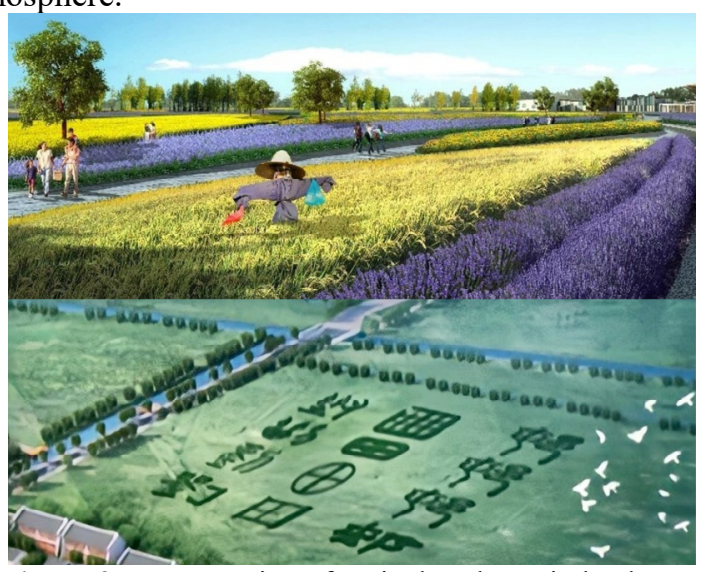

Figure 2. Construction of agricultural matrix landscape

(2) Road corridor optimization. On the basis of retaining the existing road skeleton of the village, further improve the road system, including accessibility improvement, soil road hardening and so on. All earth roads will be renovated into cement roads and characteristic brick and stone paths between houses, and several new internal roads will be built to facilitate residents' work and travel. Strengthen the vegetation coverage of the road corridor, improve the road greening system, strengthen the vegetation coverage and level, reduce pollution and noise. Improve the road landscape.

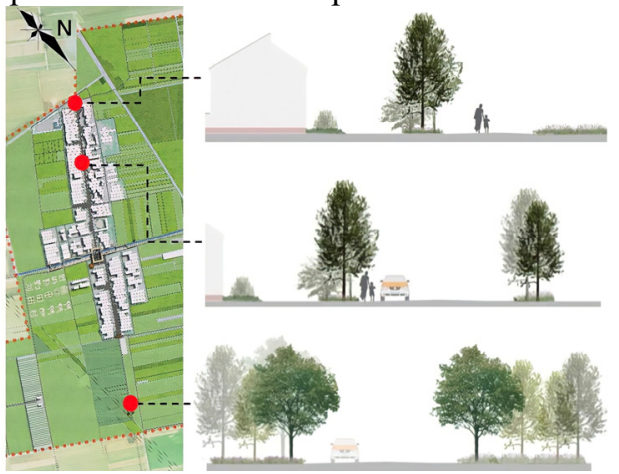

Figure 3. Road corridor greening landscape construction

(3) Waterfront corridor optimization. LiuTian village has a canal of about 1000 meters, which is lined with full section concrete. Although this kind of canal solves the problems of irrigation and surface drainage, it virtually blocks the passage of biological migration, hinders the drainage of waterlogging in farmland, and damages the quality of cultivated land. In order to reconstruct the existing canal, the ecological lining method is adopted, that is, a certain ecological hole is reserved on the slope protection brick when lining, the design water level is taken as the dividing line, the slope protection brick is lined below, and the turf slope protection is used above. Ecological lining can not only provide habitat for aquatic animals, but also facilitate the migration and survival of various aquatic animals. At the same time, the reserved ecological hole can also eliminate the water inrush in farmland and prevent soil salt inrush. And the ecological lining canal greatly reduces the use of concrete, more economic, low-carbon and environmental protection. The remaining space on both sides of the canal shall be afforested with shrubs or herbs. The revetment ecology of LiuTian village river is also poor, with soil as the bank and weeds, which is optimized by the way of "regulation + promotion". First of all, the water environment and landscape improvement, through the removal of silt, garbage, planting resistant slope plants and water purification plants and other means to solve the pollution, silt problems, and gradually improve the ecological quality of the water body; second, the combination of hard revetment and ecological revetment is adopted to coordinate the spatial scale and environmental style of water landscape and construction landscape; and then, through the establishment of Finally, part of the space is equipped with small leisure square, hydrophilic platform, fishing Pavilion and other leisure and entertainment facilities. 


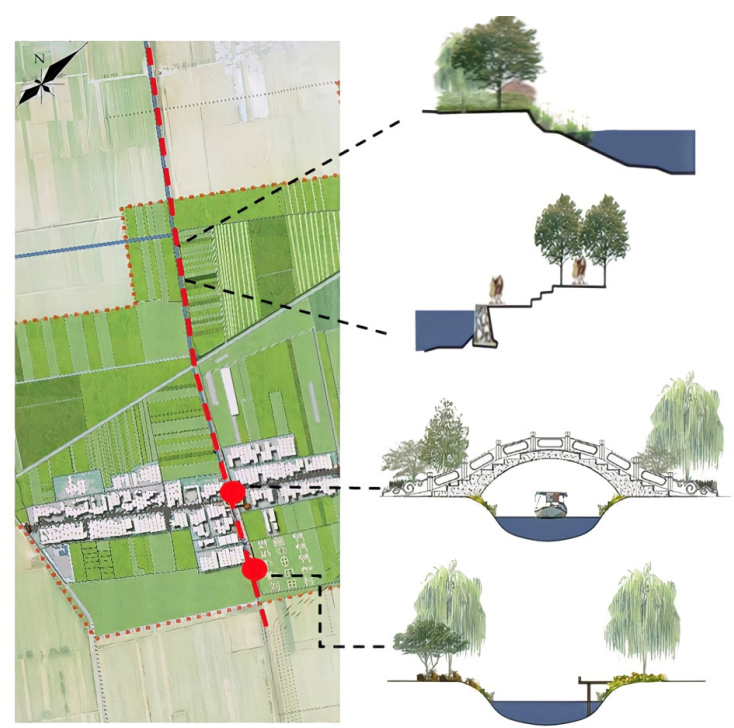

Figure 4. Waterfront corridor landscape construction

(4) The design of ancient road and compound road. Relying on the Guanzhong ancient road site in the east of the river, the ancient road culture axis embodying LiuTian village culture is constructed, and its planning and design focus on the design of ancient road and compound road. The ancient road is to restore and open an underground corridor on the basis of the original site, and use VR and other high technologies to restore and reproduce the scenes of the ancient Silk Road, especially when passing through Guanzhong of Shaanxi Province, so as to present the splendid culture of the ancient capital and the pioneering spirit of the Han nationality to the world. Taking advantage of the unique charm of the canal, a zigzag compound road is set on the ground. As an important traffic link between the two sides of the canal and the commercial street and the ancient road, the compound road can not only overlook the pastoral scenery and feel the customs of Guanzhong, but also have a short rest or enter the underground ancient road. The structural design of the compound road not only adds the elements and materials of Guanzhong architecture, but also has a sense of hierarchy and openness in space It not only increases the interest and experience, but also keeps the heavy sense of history. The ancient road and the compound road complement each other, giving people visual impact and cultural infection.

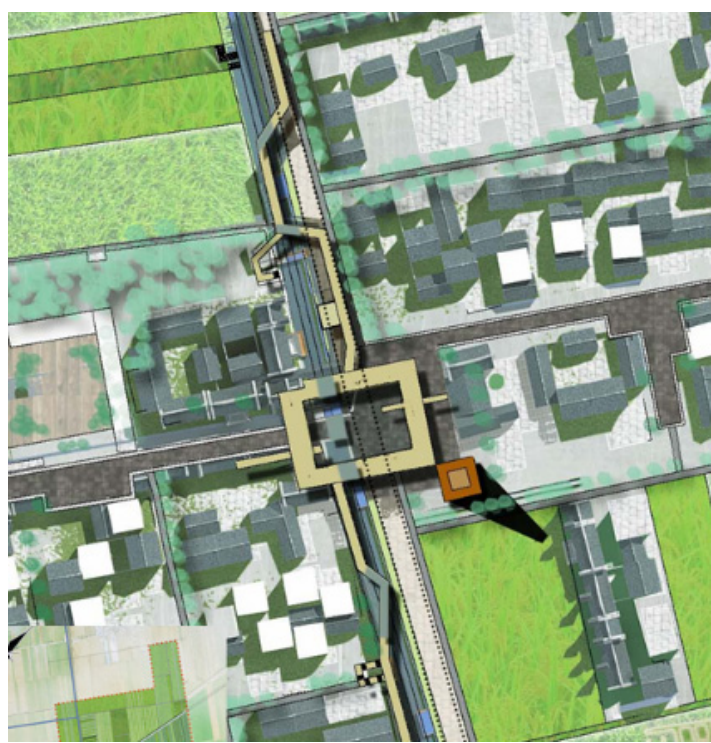

Figure 5. Plan of water system and compound road

(5) Facilities system and industry upgrading. The rural sewage ecological treatment system with septic tank, intercepting well, integrated treatment equipment and waterlogging tank as the main technical points was constructed. According to the actual situation in rural areas, the whole process from rainwater collection system to drainage system, treatment system and recycling system adopts ecological, economic and effective solutions. Through improving and reusing the septic tank and rainwater well, the ecological collection system can manage the rainwater and sewage in a unified way, and prevent the rainwater and sewage from the source. The ecological drainage system adopts the intercepting combined drainage system, and the suitable intercepting wells are selected to ensure the environmental benefits. The integrated underground sewage treatment equipment is adopted in the ecological treatment system, and a / $\mathrm{O}$ process is adopted as the main process after comprehensive consideration. Its core is to use the improved inner electrolytic packing technology to transform the traditional process, so as to improve the efficiency of sewage treatment and reduce the investment cost of equipment. The ecological recovery system uses the original waterlogging pool, integrates the plant, environment and human and other aspects to play the multiple functions of the treated water body.

\section{Conclusion and discussion}

Taking LiuTian village in Weinan City of Shaanxi Province as an example, this paper constructs the rural landscape planning method system and specific planning scheme based on the theory of environmental valueadded. Based on the results of landscape pattern analysis and comprehensive evaluation of LiuTian village, the landscape spatial network of LiuTian village was constructed, and the overall construction was carried out. The optimized and constructed landscape spatial network and layout of LiuTian village were obtained. Including matrix improvement, corridor system optimization, patch 
node beautification, facilities and industry optimization. Thus, in the five aspects of "matrix corridor patch facility", the productivity, life, ecology and landscape effect of the village landscape are improved, the landscape comprehensive index is improved, and the value-added development is realized, which provides valuable reference for the rural landscape layout.

\section{References}

1. Iiyama N, Kamada M, Nakagoshi N. Landscape \& Urban Planning. 70 (2005)

2. Assumma V, et al. Smart Innovation Systems and Technologies, Calabro F, Dellaspina L, Bevilacqua C, 101(2019)

3. Sung D G, et al. Landscape \& Urban Planning. 56(2001)

4. Kovacs F K, Nagy G G, Kollanyi L. E. Applied Ecology \& Environmental Research. 10(2012)

5. Liu Jingchao. Chang'an University (2017)

6. Liu binyi, Wang Yuncai.Chinese landscape architecture. 77(2002)

7. Xiao $\mathrm{He}$, Li liangtao, Zhang $\mathrm{Xi}$, et al. Chinese Journal of ecological agriculture. 12(2013)

8. Xie Hualin, Liu liming, $\mathrm{Xu}$ Wei. Economic geography. 03(2003) 